\title{
Competitividad Urbana: importancia, modelos y bases para su medición. Hacia un consenso conceptual para los nuevos tiempos
}

Micaela Camacho*

Universidad Católica del Uruguay mcamacho@ucu.edu.uy
Revista Cultura Económica

Año XXXVIII $\bullet \mathrm{N}^{\circ} 100$

Diciembre 2020: 13-38

DOI: https://doi.org/10.46553/cecon.38.100.2020.p13-38

Resumen: El hecho de que las concentraciones urbanas son clave en el desarrollo económico y social no puede obviarse. En esta línea, el estudio de la competitividad a nivel de ciudades y el diseño de estrategias para su impulso, son entendidos como tareas esenciales por muchos académicos y hacedores de política a nivel regional y urbano. Este diseño estratégico involucra la necesidad de alcanzar mediciones que apoyen la toma de decisiones y que, para poder obtenerse, necesitan de alguna base o modelo teórico subyacente que guíe la construcción de indicadores y la identificación de variables sobre las que recabar datos. En este sentido, este artículo tiene como eje central el estudio de los modelos sobre competitividad regional y, específicamente, sobre competitividad urbana que se han propuesto en la literatura académica en los últimos años, con el objetivo de identificar elementos sobre los que pueda existir cierto consenso, de manera de asistir a la conformación de una base teórica que sirva de herramienta para la elección (o, inclusive, la construcción) de un modelo que guíe la generación de mediciones de competitividad.

Palabras clave: Competitividad urbana; Modelos de competitividad; Ciudades

Urban Competitiveness: Importance, models and bases for its measurement. Towards a conceptual consensus for the new times

Abstract: Urban concentrations are key to economic and social development. In this line, the study of competitiveness at the city level and the design of strategies aimed to its enhancement is understood as an essential task by many academics and policy makers both at the regional and urban level. This strategic design involves the need to have measures that support the decision-making process and that are developed following a coherent theoretic framework or model. In this sense, this paper is concerned with the study of the competitiveness models at the regional and urban level proposed by the academic literature over the last years, with the central objective of finding elements over which some consensus could be identified in order to assist on the definition of a theoretical foundation that ultimately could be used as a tool to select -or even construct- a competitiveness model to guide competitiveness measures.

Keywords: Urban competitiveness; Competitiveness models; Cities

* Recibido: 19/09/2020 - Aprobado: 09/12/2020 


\section{Introducción, objetivos y metodología}

La importancia de las ciudades como eje de desarrollo económico es un concepto que, lejos de ser nuevo, ha venido tomando fuerza en la literatura económica en las últimas décadas. Inclusive, según argumenta Cheshire (2006: 1232): "las ciudades son la innovación más fundamental del desarrollo humano en los últimos 10.000 años". Las ciudades fueron el punto de partida para el desarrollo de la división del trabajo, que impactó en el aumento de la productividad y el crecimiento económico. El crecimiento de las ciudades generó necesidades que, al ser cubiertas, hicieron surgir y desarrollar insumos que apuntalaron el desarrollo de la civilización humana (medios de transporte, infraestructura física, drenajes, acceso a servicios públicos, tecnologías de comunicación, etc.). Es argumentado que las aglomeraciones urbanas contribuyeron al aumento del bienestar no solamente por la reducción de costos y el aumento de la producción, sino por la oferta de mayores opciones de consumo, menores precios y barrios especializados. Desde que nacieron, las ciudades han ido evolucionando en forma y funcionamiento (Cheshire, 2006).

Luego de un período de estancamiento, donde las ciudades solían verse como un mal resultado o residuo de la forma de organizar la economía industrial de los siglos XIX y principios del XX, la visión sobre las ciudades ha cambiado. Hoy son vistas como lugares creativos y atractivos en los que vivir y trabajar, en el marco de lo que se ha denominado "la nueva sabiduría convencional" - "the new conventional wisdom" - (Gordon y Buck, 2005). Esta nueva "sabiduría convencional" pone en relieve la importancia de las externalidades o economías de localización y el valor de las relaciones "cara a cara”, elevando por tanto el valor de los activos urbanos de toda clase. En este sentido, para la nueva sabiduría convencional no solamente las ciudades han recobrado fuerza, sino el valor de cada uno de sus activos, sus fortalezas y cualidades particulares. Al respecto, Begg (2002) plantea que, en contraste con épocas anteriores donde la tierra o los recursos minerales eran la fuente de riqueza principal, hoy son las actividades urbanas la base de la prosperidad económica. La "nueva sabiduría convencional" que surge en el debate académico apunta en la dirección de que son las ciudades y regiones los pilares fundamentales de una sociedad acelerada, globalizada y basada en la innovación (Ache y Andersen, 2008).

En la misma línea, Rogerson (1999) plantea que, asociado a la globalización de la economía, las ciudades se están convirtiendo en agentes críticos del desarrollo económico. Al respecto, argumenta Jon Azúa que, en 
el nuevo escenario económico, las ciudades serán "el centro de operaciones de ideas, creatividad, innovación y liderazgo económico, aunque, en contraste, también serán centros para la concentración de pobreza, marginalización y diferencias sociales, desbalances y desigualdades" (Azúa, 2009: 76). Por este motivo, las ciudades demandan atención, y se convierten en unidades relevantes de análisis sobre riqueza y bienestar (Azúa, 2009).

Según Glaeser (2011) la densidad urbana provee el camino más claro de la pobreza a la prosperidad y, por ello, las ciudades se están expandiendo enormemente. Por su parte, Katz y Bradley (2013) plantean que la habilidad de las ciudades, o regiones metropolitanas, de alcanzar el crecimiento económico tendrá un profundo impacto en el futuro de nuestro planeta entero. Llevando el argumento a un extremo, Barber (2013), inclusive, plantea que las ciudades pueden "salvar el mundo", dado que muchos de los mayores problemas a los que se enfrentan las sociedades son tan complejos y tan multidisciplinarios que solo pueden ser abordados y resueltos a nivel urbano y no a nivel nacional. La importancia de lo "local" también es un elemento presente en el argumento de Azúa, quien introduce un enfoque que denomina "glokal", que indica es "mucho más que el mero resumen o distinción entre los factores locales y globales" (Azúa, 2009: 23). De acuerdo a este enfoque, lo local y lo global se encuentran en el factor (la letra) "K", que actúa como conector en un mundo globalizado, en el que los factores locales son relevantemente diferenciadores. Esta letra $\mathrm{K}$ surge de las palabras inglesas asociadas a factores como: pensamiento estratégico (strategic thinking), el involucramiento de actores (stake-holding), espacios clave (key spaces), tejido de redes (knitting), conocimiento (knowledge), entre otros. El enfoque de Azúa (2009) pone en relieve la importancia de los elementos locales asociados a los factores antes mencionados, que tienen un rol central en la nueva economía globalizada.

Sin pretender elevar el argumento de la importancia de las ciudades a un extremo, no puede obviarse el hecho de que las concentraciones urbanas son muy importantes en el desarrollo económico y social. De acuerdo a estimaciones de población de la Organización de las Naciones Unidas, a nivel global más de la mitad de la población vive en áreas urbanas y se espera que para 2050 este número ascienda a casi el 70\% (United Nations, 2018). Específicamente en Latinoamérica y el Caribe el $81 \%$ de la población reside en ciudades. En el informe citado, se argumenta que el desarrollo sostenible depende de la administración efectiva del crecimiento urbano. En 
este contexto, el estudio de la competitividad urbana y sus determinantes se vuelve entonces, una temática relevante.

En la misma línea, en muchas ciudades (y regiones), las autoridades locales han involucrado a la competitividad como parte clave de sus agendas políticas de desarrollo económico. Las autoridades locales se han comenzado a preocupar por saber cómo sus economías locales se comparan con otras, no solamente en la misma región, sino con otras en todo el mundo. Diseñar estrategias para mejorar la competitividad de las localidades es ahora entendido como una tarea principal por muchos hacedores de política a nivel regional y urbano (Martin, 2006; Martin y Sunley, 2011).

Este diseño estratégico, realizado con el objetivo de mejorar la competitividad local o, más específicamente, la competitividad urbana, implica poder alcanzar mediciones que apoyen la toma de decisiones. Estas mediciones, a su vez, para poder ser realizadas necesitan de alguna base $o$ modelo teórico subyacente que guíe la construcción de indicadores y la identificación de variables sobre las que recabar datos. En este sentido, este artículo tiene como eje central el estudio de los modelos sobre competitividad regional y, específicamente, sobre competitividad urbana que se han propuesto en la literatura académica en los últimos años, con el objetivo de identificar elementos sobre los que parezca existir cierto consenso entre los investigadores de manera de asistir a la conformación de una base teórica que sirva de herramienta para la elección (o, inclusive, la construcción) de un modelo que guíe la generación de mediciones de competitividad. Para el cumplimiento del objetivo, en la siguiente sección, y como preámbulo al relevamiento de los modelos existentes, se plantean algunas consideraciones teóricas identificadas en la literatura sobre medición de competitividad. En la tercera sección se aborda el tema de la modelización de la competitividad iniciando, en primer lugar, con la revisión de antecedentes de medición a nivel regional para luego pasar específicamente a los modelos de competitividad urbana. Esta revisión de antecedentes permite identificar un patrón común en la utilización -de forma más o menos explícita o directa- del modelo de tres factores (insumoproducto-resultado), a partir del cual la competitividad urbana es vista como un proceso a través del cual se convierten ciertos elementos (insumos) en resultados intermedios (productos) que pueden ser luego utilizados para lograr resultados finales (resultados) generalmente asociados al aumento de bienestar o calidad de vida de la población de la ciudad. A partir de estos 
hallazgos, en la cuarta sección, se resumen los resultados identificando puntos en común en los distintos modelos, así como eventuales puntos de divergencia, aplicando para ello el enfoque del modelo de tres factores a cada uno de los modelos de competitividad urbana analizados.

\section{La medición de la competitividad}

En su investigación sobre modelos y mediciones de competitividad urbana, Greene et al. (2007) argumentan que los intentos de medir y comparar la competitividad de las ciudades podrían representar una carrera sin sentido en la que los ganadores y perdedores se mantienen estáticos en el tiempo (esto es comprobado en el estudio de Martin (2005), donde se puede apreciar que las 10 mejores regiones casi no muestran cambios en un período de 15 años). Si bien argumentan la utilidad que pueden tener estas medidas para los hacedores de políticas, también levantan preocupaciones respecto a cómo dichas políticas son decididas y de qué manera los recursos para "apoyar" el desarrollo de ciertas ciudades son distribuidos. Green et al. (2007) no niegan que las ciudades sean un ámbito por excelencia para el desarrollo de la innovación y el crecimiento económico, pero sí levantan dudas sobre la utilidad de comparar ciudades sin tener criterios establecidos.

$\mathrm{Al}$ respecto, Martin (2005) plantea que para que una comparación a nivel regional, que tenga por objetivo mejorar el crecimiento económico y el desempeño, tenga solidez y aplicabilidad deben tenerse en cuenta al menos tres elementos interrelacionados: (i) la selección de las regiones que "compiten" (el conjunto de comparación); (ii) la elección de los indicadores de competitividad y desempeño usados como base de comparación; (iii) cómo los resultados de la comparación regional van a ser usados para política (Martin, 2005: 30).

Con relación al primer punto propuesto por Martin, sobre la selección del grupo de contraste, Harris (2007) plantea que es necesario entender las características propias de cada ciudad para saber con quién "compite" o con quién debe compararse. Este autor pone de manifiesto que la mayor debilidad de los rankings de ciudades es que no se considera el "carácter único de cada ciudad" (Harris, 2007: 12). Por su parte, Cabrero, Orihuela y Ziccardi (2003) también plantean que la comparación debe hacerse entre ciudades similares, con estructuras económicas y problemas similares. 
A partir de lo expuesto, cabría decir, entonces, que al elegir el conjunto con el que una región (ciudad) va a compararse, podrían existir dos posiciones al respecto. Por un lado, se podría estar interesado en comparar una determinada región (ciudad) con aquella que mejor se desempeña, sin importar las diferencias en las estructuras económicas, ya que puede fijarse su crecimiento o prosperidad como objetivo (comparación-meta). Por otro lado, podría ser interesante comparar con regiones (ciudades) con estructuras industriales similares o problemas económicos similares, de forma de poder identificar "mejores prácticas" (comparación-rango).

De todas maneras, es importante mencionar que no hay una estrategia que sirva a todas las urbanizaciones por igual para mejorar la competitividad. Diferentes ciudades van a enfrentar diferentes situaciones y diferentes problemas y, por lo tanto, van a requerir distintas intervenciones de política. Ser competitivo, no significa que se tenga que contar con las mismas bases sectoriales, tecnológicas o de conocimiento que otras regiones que se desempeñan mejor (Martin, 2005). Sin embargo, una comparación inteligente y cuidadosa puede servir para identificar posibles fortalezas y debilidades sobre las que trabajar. El imperativo económico central es competir y construir competitividad a través de la búsqueda de nuevas oportunidades y fuentes de ventajas competitivas distintivas, y no "simplemente" siguiendo la mayor eficiencia en los mercados establecidos (Buck, Gordon, Harding y Turok, 2005). En este sentido, cada ciudad debe encontrar cuál es la mejor estrategia que se adapta a sus características y la comparación, entonces, puede usarse para analizar potenciales caminos y evitar posibles fallas, pero no para replicar buenas prácticas sin la adecuada adaptación.

Por otro lado, además de la elección del grupo de contraste (según se desee, comparación meta o comparación rango), y de acuerdo a los elementos que es necesario tener en cuenta según Martin (2005) para alcanzar conclusiones sólidas y aplicables, poder cotejarse con otras ciudades requiere establecer primero un mecanismo de comparación. En otras palabras, si se desea evaluar la competitividad de una ciudad, es necesario trabajar con un modelo conceptual sobre competitividad que pueda operacionalizarse para eventualmente arribar a mediciones.

En la siguiente sección se realizará una revisión analítica de los antecedentes de modelos de competitividad a nivel regional $y$, específicamente, a nivel urbano, así como la identificación de elementos 
comunes entre ellos. De esta forma, se pretende poder asistir a la construcción de una base teórica común que siente los fundamentos para la elección de modelos ya existentes o construcción de nuevos modelos a utilizar en las mediciones de competitividad.

\section{La modelización del concepto de competitividad urbana}

Aunque existe falta de consenso sobre la definición específica del concepto de competitividad ${ }^{1}$, en los últimos años se han desarrollado numerosos modelos, tanto para conceptualizar como para medir la competitividad. Según plantea Fagerberg, esto se debe a que "no es un término inventado por los teóricos sino por las personas dedicadas a la práctica, cercanas al proceso de construcción de políticas" (Fagerberg, 1996: p. 2). A este respecto, por ejemplo, las famosas mediciones anuales tanto del Foro Económico Mundial con el "Global Competitiveness Index" (World Economic Forum, 2018) y del Institute for Management Development (IMD) con el "World Competitiveness Yearbook" (IMD, 2018), son índices creados para intentar evaluar la competitividad a nivel de países, que captan anualmente la atención de los medios, de los políticos y de los constructores de política pública. Sin embargo, la naturaleza diversa y fragmentada de los estudios existentes no ha permitido consolidar una base teórica sustantiva y sólida que brinde fundamentos que soporten análisis válidos y metodologías aptas para la medición (Huggins et al., 2013).

A nivel regional y/o urbano, existen también, numerosos esfuerzos para medir el nivel competitivo. A grandes rasgos, y siguiendo las bases del relevamiento realizado por Cabrero et al. (2003), pueden distinguirse dos grandes grupos de intentos de medición, que pueden considerarse que continúan, como categorización, vigentes a la fecha².

i. Por un lado, pueden encontrarse índices realizados por consultoras privadas o algunas instancias puntuales de gobiernos regionales, cuyo objetivo principal es la comparación para el aporte a la orientación de la inversión privada, el uso de servicios, la localización de las firmas o el direccionamiento de las políticas públicas. En este grupo pueden citarse los índices de: Mercer (Quality of living); Resonance (Best Cities); Institute for Urban Strategy - The Mori Memorial Foundation (Global Power City Index, GPCI); AT Kearney (Global Cities Report); The Economist (Hotspots 2025), entre otros. En líneas generales, este tipo de índices no tiene un modelo teórico consolidado y explicitado de construcción de competitividad detrás de la medición, sino que, en 
base a una definición dada o adquirida del concepto, evalúan cada una de las ciudades/regiones, en términos de un conjunto de indicadores.

ii. Por otro lado, se encuentran los estudios con objetivos principalmente académicos. Estos estudios elaboran mediciones o proponen modelos de conceptualización de la competitividad desde una perspectiva de investigación con el fin de aportar conocimiento al área y de aplicación para políticas públicas.

Este segundo grupo de estudios es el que adquiere relevancia para los objetivos de la presente investigación. Si bien las mediciones del primer grupo aportan conocimiento en el área, en líneas generales, no poseen discusión conceptual ni aportes de modelización al concepto de competitividad (salvo casos específicos). Por lo tanto, la revisión de la literatura se centró en los estudios de carácter académico.

\section{Antecedentes generales a nivel regional 3}

De acuerdo a Leal (2015), quien realiza una revisión de los distintos modelos de competitividad regional, en general, todos los modelos subyacen a un modelo fundamental constituido por insumos, productos y resultados, en donde no solamente existe una relación entre las tres categorías, sino que hay un proceso de retroalimentación en el que los resultados se convierten posteriormente en insumos. En la misma línea, Huggins et al. (2013) plantean que los ejercicios de comparación de competitividad a nivel regional (principalmente considerando aquellos realizados para Europa y Norte América), explícita o implícitamente incorporan factores de insumos (inputs) asociados al proceso competitivo, factores de producto (outputs) asociados al desempeño competitivo y factores de resultado (outcome) asociados con el bienestar de la población. Para mostrar este marco conceptual, elaboran una adecuada ilustración de las bases del modelo subyacente, replicado en la Figura 1. 
Figura 1. Estructura del modelo de competitividad de tres factores

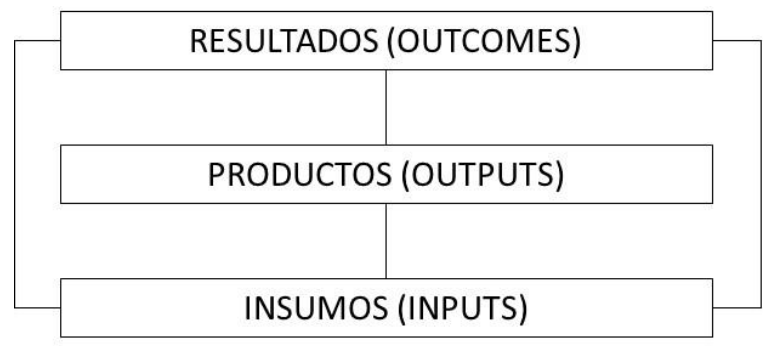

Fuente: Adaptación en base a Huggins et al. (2013)

De acuerdo a Huggins et al. (2013), los insumos (inputs) son principalmente los factores de producción que generan bienes y servicios e impulsan la actividad económica para la obtención de productos (outputs) o resultados intermedios. Ni insumos ni productos son fines en sí mismos, sino que proveen los medios para alcanzar los resultados a largo plazo. En otras palabras, los productos son los resultados directos que se obtienen de los inputs y los resultados (outcomes) son aquellos que se obtienen en el largo plazo en la forma de estándares de vida crecientes caracterizados por un desempleo decreciente y un ingreso real en aumento. A este modelo los autores lo denominan "modelo de tres factores": insumos-productosresultados 4 .

Existen algunos estudios que adoptan, para modelizar la competitividad regional, la "estructura de pirámide" (Filo, 2007; Gardiner, Martin y Tyler, 2004; Ketels, 2016; Lengyel, 2004; Lengyel y Lukovics, 2006). Haciendo un análisis de ellos, puede decirse que, de alguna manera y aunque no lo expliciten, estos modelos siguen el esquema subyacente del modelo de tres factores (inputs-outputs-outcomes). Así, por ejemplo, la estructura del modelo de pirámide para la conceptualización de la competitividad regional fue estudiado por Lengyel (2004), presentando una estructura de cuatro "capas" o "niveles", representados en la Figura 2. El primero, la base, está formado por condiciones sociales y ambientales que explican la competitividad y se pueden desarrollar o mejorar en el largo plazo (determinantes de éxito). El segundo nivel, está formado por factores que mejoran la competitividad, que se desarrollan en el corto plazo (factores de desarrollo). El tercer nivel está formado por aquellas categorías que "miden" la competitividad incluyendo: ingreso, productividad del trabajo, empleo y apertura (categorías básicas). El pico de la pirámide está destinado 
al objetivo último: estándar de vida y bienestar de la población de una región (objetivo). Si miramos esta estructura presentada por Lengyel (2004), y haciendo una comparación con el modelo de tres factores, podríamos decir que los primeros dos niveles corresponden a inputs, el tercero a outputs y el último (el pico) a outcomes.

\section{Figura 2. Estructura del modelo de pirámide de la competitividad regional}

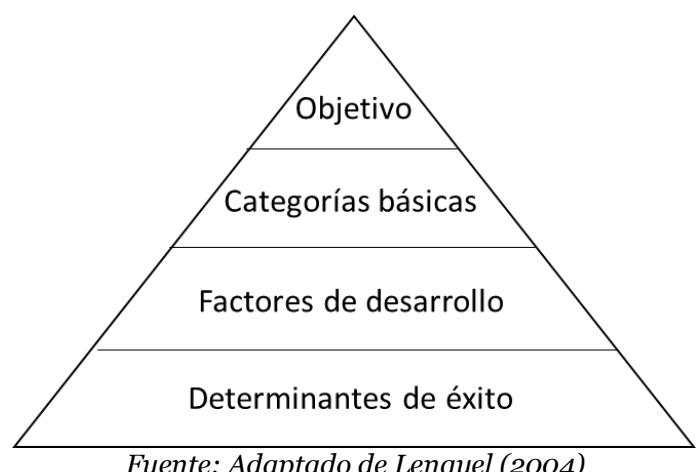

En base a este modelo piramidal, Gardiner et al. (2004) presentan una nueva versión de la "pirámide" de la competitividad regional, donde los resultados son la calidad de vida o estándar de vida (llamada por ellos "resultado objetivo"), los productos son el desempeño regional medido por el producto bruto regional, la productividad del trabajo y la tasa de empleo (lo que llaman la competitividad revelada), y los insumos varían entre el desarrollo de investigación y tecnología, la actividad de inversión extranjera, las instituciones, estructura social, cultura regional, etc., que llaman fuentes de competitividad. La estructura del modelo puede verse en la Figura 3. Entre otros estudios que utilizan la estructura de pirámide se destacan las investigaciones de Lengyel y Lukovics (2006) para el caso de regiones en Hungría, o la investigación de Filo (2007) para la inclusión de los factores humanos en la medición de la competitividad territorial en la Unión Europea. Asimismo, se considera relevante mencionar que, a nivel país, el Consejo Nacional de Competitividad de Irlanda basa su marco de trabajo en un modelo de competitividad piramidal desde hace más de dos décadas (Ketels, 2016; NCC, 2018). 


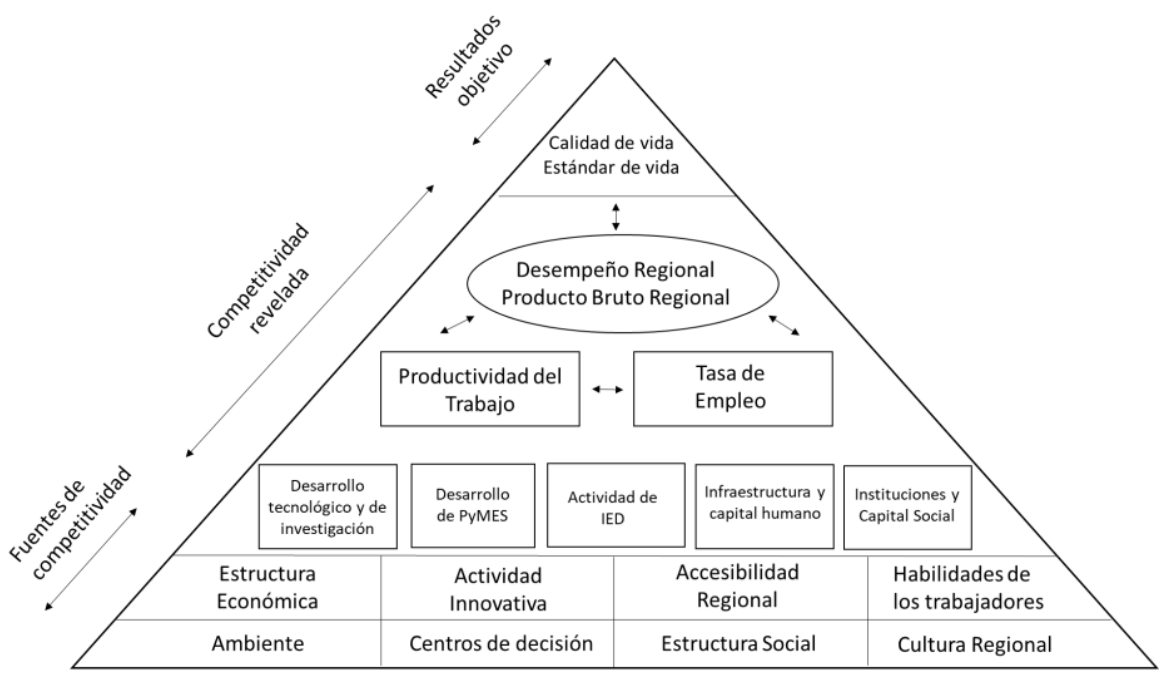

Fuente: Adaptado de Gardiner et al. (2004)

Por otra parte, un modelo que vale la pena mencionar a nivel regional es el de Martin (2003) que, aunque por su "forma" 5 no sea un modelo estrictamente piramidal, mantiene una estructura con capas e identifica una serie de niveles que conducen a un objetivo final. El modelo señala cuatro niveles en la conceptualización de la competitividad: determinantes de la competitividad regional, capacidades regionales, productos regionales y resultados regionales. Llevándolo al modelo insumos-productos-resultados (modelo de tres factores), los determinantes y las capacidades regionales serían ambos insumos. La gran diferencia que existe entre el modelo de Martin y los vistos anteriormente es que no se presenta el bienestar como el resultado último de la competitividad. Para este autor, el resultado está dado por el PBI por persona empleada, que podría catalogarse como un resultado intermedio y no como el resultado último del proceso competitivo, en línea con otros estudios mencionados previamente.

Además de los estudios mencionados, existen otras investigaciones sobre competitividad regional que siguen este modelo de tres factores de manera implícita, entre los que se pueden citar: Aiginger y Firgo (2015); Aranguren et al. (2010); Aranguren, Magro, Navarro y Valdaliso (2012); Bronisz, Heijman y Miszczuk (2008), Martin (2005). Vale mencionar que no se citan aquí los trabajos que siguen este modelo, pero cuya unidad de 
análisis es la ciudad puesto que estas investigaciones serán abordadas específicamente más adelante.

Otros estudios, aun teniendo en cuenta la existencia de factores que promueven la competitividad y factores de resultados competitivos, al momento de hacer la medición realizan el armado de los indicadores sin hacer distinción entre indicadores de inputs e indicadores de outputs. Como ejemplo de estos trabajos se pueden citar los artículos de: Annoni y Dijkstra (2013); Annoni y Kozovska (2010); Benzaquen, Carpio, Zegarra y Valdivia (2010); Dijkstra, Annoni y Kozovska (2011); Nikolic, Filipovic y Pokrajac (2016). En lo que respecta a este tipo de estudios, lo que observa la literatura es que, con el paso del tiempo, se ha movido el foco de análisis desde indicadores meramente de inputs (costos y productividad) pasando por indicadores de calidad (valor agregado, innovación, educación, etc.), llegando a indicadores de resultado que involucran objetivos más amplios que el PBI (Aiginger y Vogel, 2015). Posiblemente esto se deba a la necesidad de incorporar la nueva realidad económica, puesto que se ha dado un cambio en la manera en la que las regiones y ciudades "compiten". Como plantea Martin (2005), la competencia entre las regiones (y ciudades) ha evolucionado considerablemente en las últimas dos décadas, alejándose de los "infructuosos intentos de ofrecer los menores costos a los inversores prospectivos y a los inmigrantes" (Martin, 2005: 42). Sobre la manera en la que la competencia entre lugares ha evolucionado en el tiempo se centra la investigación de Malecki (2004), distinguiendo entre tipos de competencia "low road" basada en costos (bajos salarios, trabajo dócil y bajos impuestos) y competencia "high road" basada en el conocimiento y la innovación. En este sentido, es lógico que las comparaciones entre los lugares y por lo tanto las mediciones y modelos de competitividad hayan variado su eje a lo largo del tiempo.

A partir de este marco general, se pondrá foco, a continuación, en los modelos de competitividad a nivel de ciudades.

\section{Antecedentes a nivel de ciudades}

En lo que respecta específicamente a los modelos de competitividad urbana, es también preponderante el uso del modelo de tres factores. Esto fue corroborado por Greene et al. (2007) quienes, luego de realizar una revisión de 22 distintos modelos de conceptualización de la competitividad urbana para regiones urbanas o estados de Estados Unidos, Reino Unido y Europa continental, plantean que los bloques básicos de la mayoría de los modelos 
de competitividad analizados siguen la estructura de tres factores de insumo-producto-resultado (input-output-outcome). Esto está en línea con la observación que realiza Leal (2015) para el análisis de los modelos de competitividad regional mencionada en la sección anterior. En esta sección, además de abordar los estudios más influyentes sobre la competitividad urbana, se ampliará el análisis de los modelos de forma regional, incluyendo modelos propuestos para otras zonas además de las abarcadas por Greene et al. (2007) así como temporalmente, incluyendo modelos propuestos en la última década.

Uno de los modelos más influyentes en lo que a competitividad urbana refiere, es el desarrollado por Ian Begg en 1999. Este autor propone un modelo de competitividad a nivel de ciudades llamado el "laberinto de la competitividad urbana" (Figura 4). En este modelo se muestran conceptualmente las relaciones que existen entre las diversas variables que afectan la competitividad urbana. Más allá de la complejidad de dichas relaciones, es posible identificar en el modelo de Begg los tres "niveles" o categorías del modelo de tres factores: insumos, productos y resultados. En este sentido, en este modelo, la variable que representa el objetivo último es el estándar de vida, que podría categorizarse como el resultado del proceso de competitividad. Los productos, pueden asociarse al nivel de empleo (tasa) y la productividad. Por último, los insumos, son categorizados en cuatro: (i) tendencias sectoriales, (ii) características de las empresas, (iii) ambiente de negocios y (iv) capacidad para la innovación y el aprendizaje. Este estudio, aunque presenta una conceptualización a nivel de modelo de lo que significa competitividad urbana, no realiza una estimación empírica del mismo, es decir, no procede con el paso siguiente de medir la competitividad. 
Figura 4. El laberinto de la competitividad urbana

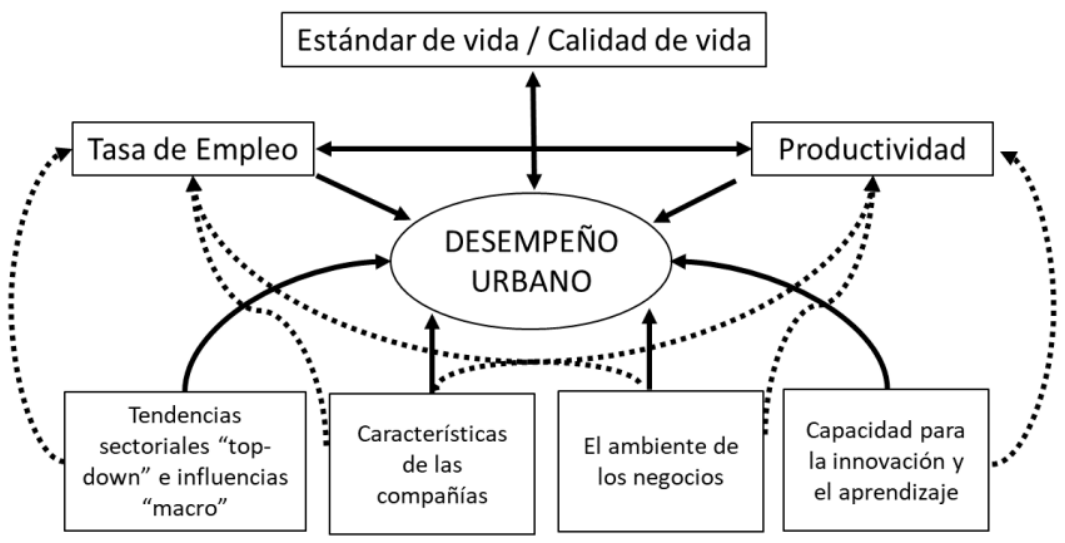

Fuente: Adaptado de Begg (1999)

En base al modelo presentado por Begg y al modelo desarrollado en forma de "pirámide"6 para la aplicación a regiones por Gardiner et al. (2004), Dudensing y Barkley (2010) aplican el modelo de pirámide a la competitividad urbana. De esta manera, en la base de la pirámide definen los inputs de competitividad; en la capa intermedia de la pirámide, lo que ellos consideran que son medidas de políticas tradicionales enfocadas en mejorar el ambiente económico a través del crecimiento industrial y que, a efectos de la comparación realizada en este estudio con el modelo de tres factores, podrían ser también inputs de competitividad. Por último, en la punta de la pirámide, incluyen los resultados competitivos que, para ellos, incluyen tanto la calidad de vida como el ingreso, el trabajo y el desarrollo sostenible. El modelo puede observarse en la Figura 5. En este sentido, surge al comparar este modelo con el de tres factores que estos autores no distinguen entre resultados y productos (outcomes y outputs). Vale mencionar que, a la hora de la aplicación empírica del modelo, que lo hacen a 151 áreas metropolitanas del sur de Estados Unidos, no replican directamente la modelización presentada en la pirámide, manteniendo los "niveles", sino que trabajan con ecuaciones que relacionan los 8 indicadores que están en la base y en el medio de la pirámide (insumos de política competitiva y ambiente para el desarrollo) y tres indicadores de outcomes/outputs o resultados del proceso competitivo (población, empleo e ingreso). 


\section{Figura 5. La pirámide de la competitividad urbana}

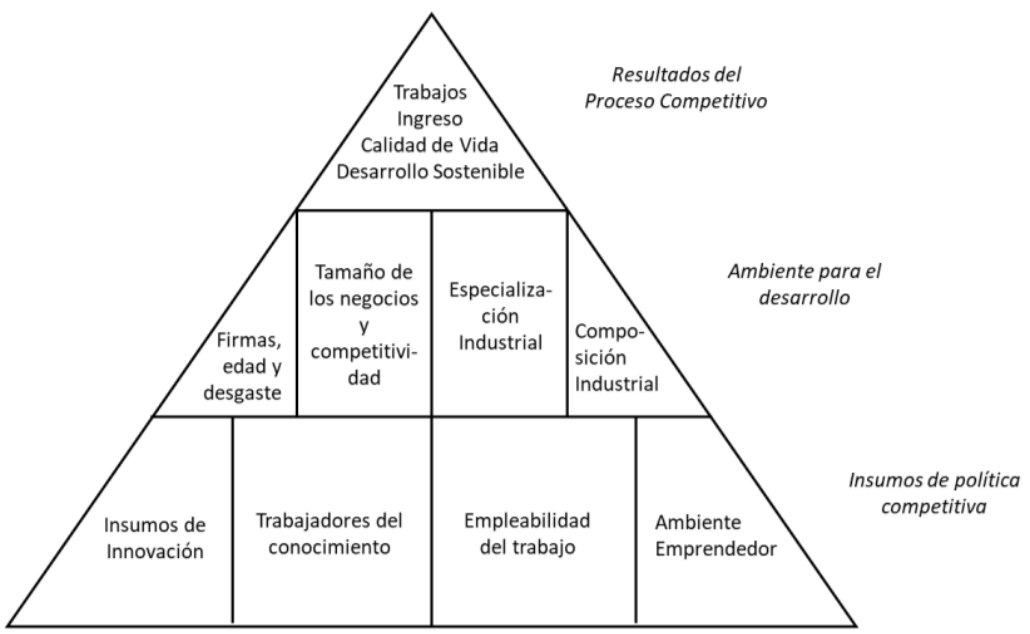

Fuente: Adaptado de Dudensing y Barkley (2010)

Por su parte, Deas y Giordano (2001) proponen un modelo de competitividad urbana donde distinguen las fuentes de competitividad (activos competitivos) de los resultados de la competitividad (resultados competitivos), el modelo puede verse en la Figura 6. Asimismo, incluyen en el modelo la relación entre estas dos categorías a través de los procesos de gobernanza, o management 7 . Estos autores no involucran el concepto de calidad de vida como resultado último de la competitividad, sino que lo evalúan como una parte más del desempeño de la ciudad. Utilizan seis variables de resultado, tres para medir la salud de las firmas y tres para medir el bienestar económico de la ciudad (las variables y los proxies que utilizan se recogen en la Tabla 1). En este sentido, podríamos decir que estos autores también agregan los productos a los resultados (agregan outputs y outcomes). Por su parte, las fuentes de competitividad (activos de competitividad) son categorizados en cuatro: (i) ambiente económico, (ii) ambiente institucional, (iii) ambiente físico y (iv) ambiente social. Las fuentes de competitividad son asimilables a los inputs en el modelo de tres factores. A la hora de computar la medición de las fuentes de competitividad urbana, Deas y Giordano toman tanto "activos" como "pasivos". Este enfoque original, implica que distinguen aquellos factores que juegan "en contra" del resultado competitivo de aquellos que juegan "a favor". Estos autores encuentran una correlación positiva entre activos (fuentes) y resultados de la competitividad para cada una de las nueve ciudades 
analizadas y sus respectivos conurbanos. Por su parte, es importante mencionar que, si bien incluyen en su modelo la importancia de los factores de gobernanza y durabilidad como parte del proceso competitivo (se pueden observar sus efectos en el modelo representado en la Figura 6), no aproximan mediciones de estos elementos. Al momento de la aplicación empírica del modelo los autores realizan mediciones de los activos competitivos (insumos) y resultados competitivos (outputs) de las 17 áreas metropolitanas de Reino Unido analizadas.

\section{Figura 6: Modelo de competitividad urbana}

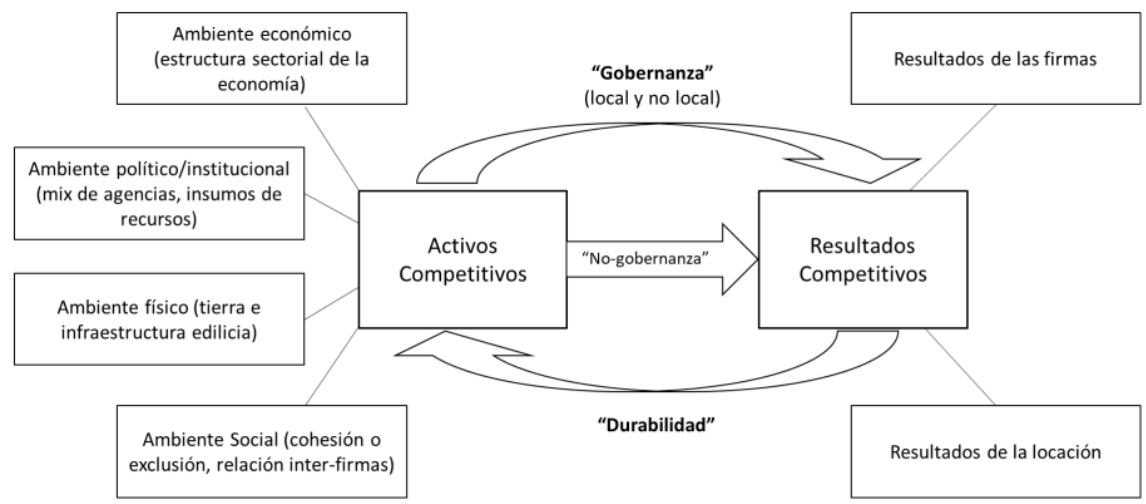

Fuente: Adaptado de Deas y Giordano (2001)

Otro modelo de competitividad urbana comparable es el planteado por Kresl (1995). Este autor propone la conceptualización de la competitividad como una función que depende de determinantes económicos y determinantes estratégicos. Los primeros, incluyen los factores de producción, la infraestructura, la ubicación, la estructura económica y las comodidades urbanas. Los determinantes estratégicos incluyen la eficiencia del gobierno, la estrategia urbana, la cooperación público-privada y la flexibilidad institucional. En definitiva, estos determinantes propuestos por Kresl (1995) pueden ser vistos como los inputs del proceso competitivo. Según plantea este autor, una de las diferencias entre ambos conjuntos es que los determinantes económicos pueden representarse por factores cuantitativos mientras que los determinantes estratégicos son de naturaleza cualitativa ${ }^{8}$. El autor da un listado de características que debe cumplir una ciudad competitiva, hace una revisión de literatura para justificar la incorporación de cada uno de los 
factores a la función competitiva (mencionados previamente) y define el tipo de datos necesarios para realizar el análisis empírico que propone abordar en futuras investigaciones.

El testeo empírico del modelo propuesto por Kresl (1995) lo realizaron Kresl y Singh en $1995^{9}$ y, posteriormente, en 1999. En este último estudio mencionado, Kresl y Singh miden la competitividad urbana de 24 áreas metropolitanas de Estados Unidos, como una combinación lineal de tres variables: crecimiento de las ventas minoristas, crecimiento del valor agregado de las manufacturas y crecimiento en la recepción de servicios empresariales. A partir de la regresión de la variable hallada, "competitividad urbana", con un listado de factores encuentran una relación significativa entre competitividad y determinantes primarios (el listado de estos determinantes se presenta en la Tabla 1). Vale mencionar que los autores trabajan solamente con los determinantes económicos, puesto que explican que no logran conseguir a tiempo los datos necesarios de carácter cualitativo para analizar los determinantes estratégicos. También vale señalar que dos de los determinantes primarios (\% de trabajadores EARM ${ }^{10}$ sobre el total de trabajadores y crecimiento en el ingreso per cápita), fueron descompuestos en ocho variables adicionales a las que llaman determinantes secundarios.

En un estudio posterior, Kresl y Singh (2012) retoman este modelo para aplicarlo a datos más recientes de 23 áreas metropolitanas de Estados Unidos. Realizan un cambio en el modelo, motivado por los cambios en la realidad económica de las últimas décadas y el reconocimiento que el peso de las manufacturas no necesariamente es un elemento clave para la competitividad (una ciudad puede elegir, como opción estratégica, no enfocarse en industrias manufactureras y ser igualmente competitiva) ${ }^{11}$. De esta manera, cambian la variable crecimiento en el valor agregado de las manufacturas por la variable crecimiento en los salarios por empleado. Al igual que en el estudio de 1999, los autores no evalúan los determinantes estratégicos y de forma similar al estudio anterior encuentran cuatro determinantes secundarios, esta vez, a partir de la variable "porcentaje de población mayor a 25 años con título de grado" (el listado de variables y proxies utilizadas se encuentran en la Tabla 1).

Otro modelo de competitividad urbano que sigue el patrón insumoproducto-resultado es el propuesto por Cooke (2004), quien realiza un índice de competitividad agregando las distintas variables de cada uno de estos tres niveles. Este autor hace hincapié en la relación entre la 
competitividad de la ciudad y la competitividad de las empresas que operan allí, argumentando que son "conceptos interdependientes" (Cooke, 2004: 154). Por este motivo, según este autor, los insumos de la competitividad son la densidad de empresas, la proporción de empresas basadas en el conocimiento y la participación total de la economía (tasas de actividad); los productos son esencialmente la productividad de la ciudad (aproximada por el PBI per cápita) y los resultados son el nivel medio de salarios y la proporción de personas buscando trabajo que están trabajando.

Por su parte, en un estudio sobre la competitividad en las ciudades mexicanas, Sobrino (2002) realiza la discusión conceptual en las líneas del modelo insumo-producto-resultado de forma implícita. De esta manera, plantea que "la competitividad se refiere a la interconexión entre el desempeño económico y el incremento en la calidad de vida de la población" (Sobrino, 2002: 329). Por otra parte, plantea que "los factores explicativos de la competitividad local son adaptaciones de los enfoques sobre ventajas competitivas de las naciones y de las proposiciones de las teorías de localización de las actividades económicas" (Sobrino, 2002: 326). De alguna manera, entonces, en la conceptualización de la competitividad presentada en el artículo mencionado puede verse como este autor habla de factores (inputs), desempeño económico (outputs) y calidad de vida (outcomes), aunque no explicita un modelo de construcción competitiva. Al momento de la medición el artículo se centra en la competitividad industrial de 30 ciudades mexicanas. Para ello, primero establece un indicador de competitividad sobre la base del desempeño de cada ciudad en cuatro variables (se listan en la Tabla 1). Vale mencionar que, si bien el autor habla sobre que el resultado último de la competitividad es el aumento de la calidad de vida, al momento de la medición (construcción del indicador de competitividad) utiliza cuatro variables indicadoras de desempeño (output) o resultado intermedio. Para el cálculo de los determinantes de la competitividad (inputs), el autor propone que los factores se agrupan en tres tipos de ventajas competitivas: empresariales, territoriales y distributivas.

Se incluye en el relevamiento un estudio de carácter teórico-empírico desarrollado por Sinkiené (2009) para la ciudad de Lituania, donde se realiza un testeo de cuáles son los factores que deben tenerse en cuenta para medir el desempeño y los inputs en una eventual medición de competitividad. Si bien este estudio no se embarca en la medición, presenta un relevamiento de factores a ser evaluados en cada nivel, realizado a través de encuestas a expertos ${ }^{12}$. Un aporte adicional de este estudio es que, 
además de los factores considerados como insumos a la competitividad (factores institucionales, factores humanos, factores físicos y factores económicos), presenta una serie de elementos que deben ser tenidos en cuenta para el análisis de la competitividad de las ciudades pero que no se determinan en ella: factores económicos (situación macroeconómica, fiscal, política, etc.); factores tecnológicos (desarrollo de TI, política de desarrollo tecnológico, etc.); factores político-legales (estabilidad político-económica, acuerdos internacionales, seguridad externa, etc.); factores socio-culturales (demografía, igualdad de género, estilo de vida, efectividad del sistema de salud, etc.); factores ecológicos (clima, recursos hidrográficos, uso de la tierra, recursos energéticos, etc.). Este grupo de factores son colocados por la autora en la base del modelo de construcción competitiva. Otro elemento interesante de la propuesta presentada por Sinkiené, es que coloca al interior del modelo el elemento de "proceso", ocupando un "nivel" adicional entre los inputs y los outputs y compuesto por cinco elementos: las personas en el centro, el ocio, el transporte, el trabajo y la vivienda.

Otras investigaciones que tienen como objetivo la medición de la competitividad a nivel de ciudades, se centran solamente en los factores o determinantes (inputs) de la competitividad, o solamente en las variables de desempeño (outputs). Como ejemplos de este tipo de estudios, en el caso de los inputs, puede citarse el estudio desarrollado por Ni et al. (2017) aplicado a ciudades a nivel global que utiliza indicadores en 6 subgrupos (conexión global, demanda local, ambiente software, ambiente hardware, elementos locales, calidad de las empresas). En el caso de los outputs, puede citarse el estudio de Jiang y Shen (2010) para ciudades de China, que utiliza tres subgrupos de indicadores: (i) competitividad económica (aproximada por el desempeño económico, la estructura y capacidad económica y la apertura de mercados); (ii) competitividad social (aproximada por los recursos humanos y la educación, la calidad de vida, el nivel de desarrollo urbano y la acción e iniciativas del gobierno) y (iii) competitividad ambiental (aproximada por la calidad del medioambiente).

Otros estudios elaboran indicadores sobre competitividad urbana, sin identificar grupos de insumos, productos o resultados, este es el caso de Bulu (2011) para el caso de Turquía, Cabrero et al. (2003) para el caso de México, Sáez y Periñáez (2015) para ciudades europeas, Zhang et al. (2015) para el caso de Chengdú en China, entre otros.

Finalmente, hay estudios que sin arribar a mediciones directas de competitividad utilizan indicadores existentes para evaluar la situación 
competitiva de distintas ciudades. Este es el caso, por ejemplo, del estudio de Bailey et al. (2002), quienes estudian la situación de dos ciudades: Edimburgo y Glasgow, a través del cambio en el PBI per cápita, empleo, especialización industrial, formación de nuevas firmas, mercados de trabajo y disponibilidad de tierra.

\section{Resultados y conclusiones}

A partir de la revisión de antecedentes sobre modelos y mediciones de competitividad urbana, y en línea con los hallazgos de estudios anteriores para competitividad regional (Leal, 2015) y competitividad urbana de Estados Unidos y Europa (Greene et al., 2007), puede decirse que, en lo que respecta a los estudios que persiguen objetivos académicos ${ }^{13}$, la gran mayoría de ellos utiliza directa o indirectamente el modelo de tres factores (input-output-outcome). Es decir, aún en aquellos estudios que no explicitan un modelo específico de competitividad, puede identificarse en su marco teórico una línea argumental que lo empareja al modelo de tres factores. A modo de resumir el análisis y mostrar cómo cada modelo analizado sobre competitividad urbana puede asimilarse al modelo de tres factores, se presenta en la Tabla 1 este emparejamiento. Se identifican, para cada uno de los factores del modelo (inputs-outputs-outcomes), las variables específicas que fueron elegidas en cada investigación estudiada y que pueden asociarse a cada nivel. Esto permite identificar puntos en común en los distintos modelos, así como eventuales puntos de divergencia a ser contemplados. Sin embargo, más allá de los eventuales puntos de divergencia, puede encontrarse en los modelos de competitividad urbana propuestos un cierto consenso entre los investigadores (a partir de la utilización que hacen explícita o implícitamente del modelo de tres factores), sobre la conceptualización de la competitividad a nivel de ciudades, que es vista como un proceso a través del cual se convierten ciertos elementos (insumos/inputs) en resultados intermedios (productos/outputs) que pueden ser luego utilizados para lograr resultados finales (resultados/outcomes) generalmente asociados al aumento de bienestar o calidad de vida de la población de la ciudad.

A partir de estos hallazgos, cabría concluir que parece existir un cierto consenso en cuanto a que la competitividad urbana es un proceso y que, para ser contemplado en su completa complejidad, deben considerarse elementos de insumos del proceso, elementos de resultados intermedios y elementos de resultados finales, asociados a aumentos de bienestar. 
Tabla 1. Modelos de competitividad urbana bajo el enfoque del modelo de 3 factores

\begin{tabular}{|c|c|c|c|c|c|c|c|c|}
\hline & \begin{tabular}{|l} 
Begg (1999) \\
Teórico
\end{tabular} & $\begin{array}{l}\text { Dudensing \& Barkley (2010) } \\
\text { Surde EEUU }\end{array}$ & $\begin{array}{l}\text { Deas \& Giordano (2001) } \\
\text { Inglaterra }\end{array}$ & $\begin{array}{l}\text { Kresl \& Singh (1999) } \\
E E U U\end{array}$ & $\begin{array}{l}\text { KresI \& Singh (2012) } \\
\text { EEUU }\end{array}$ & $\begin{array}{l}\text { Cooke (2004) } \\
\text { Inglaterra }\end{array}$ & $\begin{array}{l}\text { Sobrino (2002) } \\
\text { México }\end{array}$ & $\begin{array}{l}\text { Sinkiené (2009) } \\
\text { Teórico- Lituania }\end{array}$ \\
\hline OUTCOME & Calidad de Vida & \multirow{2}{*}{$\begin{array}{l}\text { Densidad de población } \\
\text { Densidad de empleo } \\
\text { Ingreso per cápita }\end{array}$} & \multirow{2}{*}{$\begin{array}{l}\text { Desempeño de las empresas } \\
\text { (Firmas registradas IVA/Tot. pob.; } \\
\text { Nuevas empresas/Tot. empresas; } \\
\text { Empresas cotizantes de resposabilidad } \\
\text { limitada/Tot. pob. conurbano) } \\
\\
\text { Bienestar de la ciudad } \\
\text { (PBI per cápita; Alquileres comerciales } \\
\text { e industriales; \% de residentes activos) }\end{array}$} & \multirow{2}{*}{$\begin{array}{l}\text { Crec. VA de las manufacturas } \\
\text { Crec. ventas minoristas } \\
\text { Crec. servicios empresariales }\end{array}$} & \multirow{2}{*}{$\begin{array}{l}\text { Crec. salarios por empleado } \\
\text { Crec. ventas minoristas } \\
\text { Crec. serv. empresariales }\end{array}$} & $\begin{array}{l}\text { Salario } \\
\text { Desempleo }\end{array}$ & \multirow{2}{*}{$\begin{array}{l}\text { Cbio. en la part. abs. en VBP nac. } \\
\text { Cbio. en la part. rel. en VBP nac. } \\
\text { Crecimiento absoluto del VBP } \\
\text { Base económica ( } \triangle \text { VBP/ } \triangle \text { pob) }\end{array}$} & Calidad de Vida \\
\hline OUTPUT & \begin{tabular}{|l} 
Tasa de Empleo \\
Productividad
\end{tabular} & & & & & Productividad (PBI/cápita) & & $\begin{array}{l}\text { Productividad; VA / persona; } \\
\text { Ingreso/Poder de compra; } \\
\text { Beneficios corporativos; } \\
\text { Crecimiento lugar de trabajo; } \\
\text { Inversión interna; Inmigración; } \\
\text { Imagen de la ciudad; Situación } \\
\text { ecológica }\end{array}$ \\
\hline INPUTS & \begin{tabular}{|l} 
Patrones sectoriales \\
Características de las empresas \\
Ambiente de negocios \\
Cap. innovación y aprendizaje
\end{tabular} & $\begin{array}{l}\text { Innovación } \\
\text { Disp. de trabajo calificado } \\
\text { Disp. y calid. de la fuerza trabajo } \\
\text { Actividad emprendedora } \\
\text { Edad de las empresas } \\
\text { Tamaño de las empresas } \\
\text { Especialización industrial } \\
\text { Composición industrial }\end{array}$ & $\begin{array}{l}\text { Ambiente económico }(6+/ 2-) \\
\text { Ambiente institucional }(2+/ 0-) \\
\text { Ambiente físico (1+ / 2-) } \\
\text { Ambiente social (2+ / 3-) }\end{array}$ & $\begin{array}{l}\text { Determinantes Económicos } \\
\text { (crecimiento en el ingreso oper cápita; } \\
\text { centros de investigación / VA } \\
\text { manufacturas; crecimiento\% firmas } \\
\text { con más de } 100 \text { empleados; fuerza } \\
\text { laboral con postgrado; \% trabajadores } \\
\text { EARM; crecimientonumero de } \\
\text { instituciones culturales; crecimiento en } \\
\text { el stock de capital para el estado; } \\
\text { export / producción total) } \\
\\
\\
\text { Determinantes Estratégicos } \\
\text { (N/D) }\end{array}$ & $\begin{array}{l}\text { Determinantes Económicos } \\
\text { (crecimiento en va manufacturas; } \\
\text { camas de hospital c } 1000.000 h \text { \% } \% \text { pobl. } \\
\text { de } 25+\text { con grado universitario; \% } \\
\text { empleo en finanzas, seguros o sector } \\
\text { inm; nummero de instituciones } \\
\text { culturales; \% de firmas con menos de } \\
100 \text { empleados; centros de } \\
\text { investigación/fuerza de trabajo; } \\
\text { infreastructura de transporte y } \\
\text { servicios) } \\
\text { Determinantes Estratégicos } \\
\text { (N/D) }\end{array}$ & $\begin{array}{l}\text { Densidad de empresas } \\
\% \text { negocios basados en conoc. } \\
\text { Participación económica (TA) }\end{array}$ & 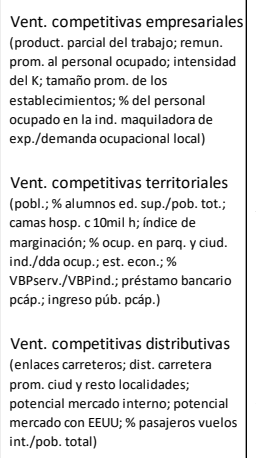 & 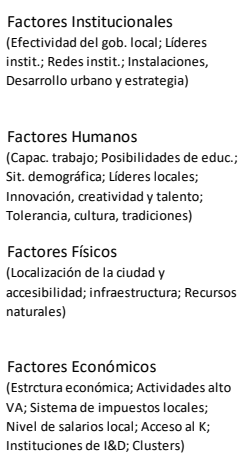 \\
\hline
\end{tabular}

Fuente: Elaboración propia 


\section{Referencias Bibliográficas}

Ache, P. \& Andersen, H. T. (2008). "Cities between competitiveness and cohesion: discourses, realities and implementation-introduction". In P. Ache, H. T. Andersen, T. Maloutas, M. Raco \& T. T. Taşan-Kok (eds.). Cities between competitiveness and cohesion, Dordrecht: Springer, 3-18.

Aiginger, K. (2006). "Competitiveness: from a dangerous obsession to a welfare creating ability with positive externalities". Journal of Industry, Competition and Trade, 6(2), 161-177.

Aiginger, K. \& Firgo, M. (2015). Regional competitiveness under new perspectives. WWWfor Europe Policy Paper No. 26. WIFO Studies, WIFO, No. https://ideas.repec.org/b/wfo/wstudy/58501.html 58501.

Aiginger, K. \& Vogel, J. (2015). "Competitiveness: from a misleading concept to a strategy supporting Beyond GDP goals". Competitiveness Review, 25(5), 497-523.

Annoni, P. \& Dijkstra, L. (2013). EU Regional Competitiveness Index: RCI 2013. Luxemburg: Publications Office of the European Union.

Annoni, P. \& Kozovska, K. (2010). EU Regional Competitiveness Index: RCI 2010. Luxembourg: Publications Office of the European Union.

Aranguren, M. J., Franco, S., Ketels, C., Murciego, A., Navarro, M. \& Wilson, J. (2010). Benchmarking regional competitiveness in the European Cluster Observatory. European Commission.

Aranguren, M. J., Magro, E., Navarro, M. \& Valdaliso, J. Ma. (2012). Estrategias para la construcción de ventajas competitivas regionales: El caso del País Vasco. Madrid: Instituto Vasco de Competitividad - Fundación Deusto \& Marcial Pons.

Azúa, J. (2009). Clusterizing and Glokalizing the Economy: The Magic of the Process (Enovatinglab Ed.). Bogotá: Editorial Oveja Negra.

Bailey, N., Docherty, I. \& Turok, I. (2002). "Dimensions of city competitiveness: Edinburgh and Glasgow in a UK context". In I. Begg (ed.). Urban competitiveness: Policies for dynamic cities, Bristol: Bristol University Press, 135-160.

Barber, B. R. (2013). If mayors ruled the world: Dysfunctional nations, rising cities. New Heaven: Yale University Press.

Begg, I. (1999). "Cities and competitiveness". Urban studies, 36(5-6), 795809.

Begg, I. (2002). Urban competitiveness: policies for dynamic cities. Bristol: Policy Press.

Benzaquen, J., Carpio, L. A. d., Zegarra, L. A. \& Valdivia, C. A. (2010). "Un índice regional de competitividad para un país". Revista CEPAL (102), 69-86.

Bristow, G. (2005). "Everyone's a 'winner': problematising the discourse of regional competitiveness". Journal of Economic Geography, 5(3), 285-304. 
Bronisz, U., Heijman, W. \& Miszczuk, A. (2008). "Regional competitiveness in Poland: Creating an index". Jahrbuch für Regionalwissenschaft, 28(2), 133-143.

Buck, N. H., Gordon, I., Harding, A. \& Turok, I. (2005). Changing cities: Rethinking urban competitiveness, cohesion, and governance. Hampshire \& New York: Palgrave Macmillan.

Buckley, P. J., Pass, C. L. \& Prescott, K. (1988). "Measures of international competitiveness: A critical survey". Journal of marketing management, 4(2), 175-200.

Bulu, M. (2011). "Measuring competitiveness of cities: Turkish experience". International Journal of Knowledge-Based Development, 2(3), 267-281.

Cabrero, E., Orihuela, I. \& Ziccardi, A. (2003). Ciudades competitivasciudades cooperativas: conceptos claves y construcción de un índice para ciudades mexicanas. Documento de trabajo, 139.

Cellini, R. \& Soci, A. (2002). "Pop competitiveness". Banca Nazionale del Lavoro Quarterly Review, 55(220), 71.

Cheshire, P. C. (2006). "Resurgent cities, urban myths and policy hubris: what we need to know". Urban studies, 43(8), 1231-1246.

Cooke, P. (2004). "Competitiveness as cohesion: social capital and the knowledge economy". In M. Boddy \& M. Parkinson (eds.). City matters: Competitiveness, cohesion and urban governance. Bristol: Policy Press, 93-109.

Deas, I. \& Giordano, B. (2001). "Conceptualising and measuring urban competitiveness in major English cities: an exploratory approach". Environment and Planning A, 33(8), 1411-1429.

Dijkstra, L., Annoni, P. \& Kozovska, K. (2011). "A new regional competitiveness index: Theory, methods and findings". European Union Regional Policy Working Papers, 2.

Dudensing, R. M. \& Barkley, D. L. (2010). "Competitiveness of Southern Metropolitan Areas: The Role of New Economy Policies”. Review of Regional Studies, 40(2), 197-226.

Fagerberg, J. (1996). "Technology and competitiveness". Oxford Review of Economic Policy, 12(3), 39-51.

Filo, C. (2007). Territorial Competitiveness and the Human Factors. Paper presented at International Conference of Territorial Intelligence, Huelva.

Gardiner, B., Martin, R. \& Tyler, P. (2004). "Competitiveness, Productivity and Economic Growth across the European Regions". Regional studies, 38(9), 1045-1067.

Glaeser, E. (2011). Triumph of the city: How urban spaces make us human. London: Pan Macmillan.

Gordon, I. \& Buck, N. (2005). "Introduction: Cities in the new conventional wisdom". In N. Buck, I. Gordon, A. Harding \& I. Turok (eds.). Changing cities: Rethinking urban competitiveness, cohesion, and governance. Hampshire \& New York: Palgrave Macmillan, 1-21. 
Greene, F. J., Tracey, P. \& Cowling, M. (2007). "Recasting the city into cityregions: place promotion, competitiveness benchmarking and the quest for urban supremacy". Growth and Change, 38(1), 1-22.

Harris, N. (2007). City Competitiveness. Originally drafted for a World Bank study of competitiveness in four Latin American cities.

Huggins, R., Izushi, H., Prokop, D. \& Thompson, P. (2014). Regional competitiveness, economic growth and stages of development. Paper presented at the Proceedings of Rijeka School of Economics.

Huggins, R., Izushi, H. \& Thompson, P. (2013). "Regional competitiveness: theories and methodologies for empirical analysis". Journal of CENTRUM Cathedra: The Business and Economics Research Journal, 6(2), 155-172.

Institute for Management Development (IMD) (2018). World Competitiveness Yearbook. https://www.imd.org/researchknowledge/books/world-competitiveness-yearbook-2018/

Jiang, Y. \& Shen, J. (2010). "Measuring the urban competitiveness of Chinese cities in 2000". Cities, 27(5), 307-314.

Katz, B. \& Bradley, J. (2013). The metropolitan revolution. Washington, DC: The Brookings Institution.

Ketels, C. (2016). Review of competitiveness frameworks. Retrieved from https://www.hbs.edu/faculty/Publication\%20Files/Review\%20of\% 20Competitiveness\%20Frameworks\%20_3905ca5f-c5e6-419b8915-5770a2494381.pdf

Kitson, M., Martin, R. \& Tyler, P. (2004). "Regional competitiveness: an elusive yet key concept?” Regional studies, 38(9), 991-999.

Kresl, P. (1995). "The determinants of urban competitiveness: a survey". In P. Kresl \& G. Gappert (eds.). North American Cities and the Global Economy. Thousand Oaks, CA: Sage Publications, 45-68.

Kresl, P. \& Singh, B. (1995). "The competitiveness of cities: The United States". In OECD (ed.). Cities and the new global economy. Melbourne: The Government of Australia and the OECD, 424-446.

Kresl, P. \& Singh, B. (1999). "Competitiveness and the urban economy: twenty-four large US metropolitan areas”. Urban studies, 36(5-6), 1017-1027.

Kresl, P. \& Singh, B. (2012). "Urban competitiveness and US metropolitan centres”. Urban Studies, 49(2), 239-254.

Lall, S. (2001). Competitiveness, technology and skills. Cheltenmham: Edward Elgar.

Leal, C. (2015). Hacia un análisis integral de la competitividad territorial: el caso del estado de Querétaro, México. (Tesis de Doctorado). Universidad de Deusto, País Vasco.

Lengyel, I. (2004). "The pyramid model: enhancing regional competitiveness in Hungary". Acta Oeconomica, 54(3), 323-342.

Lengyel, I. \& Lukovics, M. (2006). An attempt for the measurement of regional competitiveness in Hungary. Paper presented at the conference on "Enlargement, Southern Europe and the 
Mediterranean", 46th Congress of the European Regional Science Association. Volos, Greece, August 3oth-September 3 rd.

Malecki, E. (2004). "Jockeying for position: What it means and why it matters to regional development policy when places compete”. Regional studies, 38(9), 1101-1120.

Martin, R. (2003). A study on the factors of regional competitiveness, WIFO Studies, WIFO, No. 25005.

Martin, R. (2005). Thinking about regional competitiveness-critical issues. Nottingham: East Midlands Development Agency. http://irep.ntu.ac.uk/id/eprint/519/

Martin, R. (2006). "Economic geography and the new discourse of regional competitiveness". In S. Bagchi-Sen \& H. Lawton Smith (eds.). Economic Geography: Past, Present and Future. London: Routledge, 159-172.

Martin, R. \& Sunley, P. (2011). "Regional competitiveness: clusters or dynamic comparative advantage?”. In R. Huggins \& H. Izushi (eds.). Competition, Competitive Advantage and Clusters: The Ideas of Michael Porter. Oxford: Oxford University Press, 211-238.

Mulatu, A. (2016). "On the Concept of 'Competitiveness' and its Usefulness for Policy". Structural Change and Economic Dynamics, 36, 50-62.

National Competitiveness Council (NCC) (2018). Ireland's Competitiveness Challenge

2018. http://www.competitiveness.ie/Publications/2018/Competitiveness -Challenge-2018.pdf

Ni, P., Kamiya, M. \& Ding, R. (2017). "Global Urban Competitiveness: Theoretical Framework". In Cities Network Along the Silk Road. Singapore: Springer, 15-29.

Nikolić, M., Filipović, M. \& Pokrajać, S. (2016). "Regional competitiveness for achieving sustainable development: The case of Serbia". Industrija, 44(3), 7-26.

Reinert, E. S. (1995). "Competitiveness and its predecessors-a 500-year cross-national perspective". Structural change and economic dynamics, 6(1), 23-42.

Rogerson, R. J. (1999). "Quality of life and city competitiveness". Urban studies, 36(5-6), 969-985.

Sinkiene, J. (2009). "Competitiveness factors of cities in Lithuania". Public Policy and Administration, 1(29).

Sobrino, J. (2002). "Competitividad y ventajas competitivas: revisión teórica y ejercicio de aplicación a 30 ciudades de México". Estudios demográficos y urbanos, 311-361.

Sáez, L. \& Periáñez, I. (2015). "Benchmarking urban competitiveness in Europe to attract investment". Cities, 48, 76-85.

Turok, I. (2004). "Cities, regions and competitiveness". Regional studies, 38(9), 1069-1083.

United Nations (2018). World Urbanization Prospects: The 2018 Revision. 
Wilson, J. (2008). "Territorial competitiveness and development policy. Orkestra”. Basque Institute of Competitiveness. Basque Country, Spain, 31.

World Economic Forum (2018). The Global Competitiveness Report. Zhang, W., Deng, F. \& Liang, X. (2015). Comprehensive Evaluation of Urban Competitiveness in Chengdu Based on Factor Analysis. Paper presented at the Proceedings of the Ninth International Conference on Management Science and Engineering Management.

\footnotetext{
${ }^{1}$ La mayoría de la literatura académica sobre el tema competitividad argumenta que no existe aún consenso en la definición específica del término (Aiginger, 2006; Aiginger y Vogel, 2015; Aranguren et al., 2010; Begg, 1999; Bristow, 2005; Cellini y Soci, 2002; Huggins, Izushi, Prokop y Thompson, 2014; Huggins, Izushi y Thompson, 2013; Kitson, Martin y Tyler, 2004; Lall, 2001; Martin, 2003; Mulatu, 2016; Reinert, 1995; Turok, 2004; Wilson, 2008).

${ }_{2}^{2}$ Vale mencionar que, en realidad, Cabrero et al. (2003) identifican tres grupos de estudios. Sin embargo, el último grupo identificado dicen que es un mix entre los grupos anteriores y solo identifican en este grupo un estudio realizado, además, a nivel nacional. Por este motivo, no se consideró este tercer grupo en la taxonomía propuesta en la presente investigación.

$3 \mathrm{Si}$ bien la revisión de antecedentes se centra, principalmente, en aquellos estudios que realizan medición de competitividad, en algunos casos se mencionan trabajos puramente teóricos (que no arriban a una medición de competitividad) cuando se considera que enriquecen pertinentemente el análisis.

4 En adelante se utilizarán indistintamente para referirse al primer factor, las palabras inputs o insumos; para referirse al segundo factor las palabras productos, outputs o resultados intermedios; y para referirse al tercer factor, las palabras outcomes o resultados finales.

$5 \mathrm{El}$ autor lo identifica con un "sombrero" y denomina su modelo: "El sombrero de la competitividad" (Martin, 2003: 2-36).

${ }^{6}$ Para una introducción sobre los modelos de "pirámide" aplicados a la competitividad ver la sección anterior.

$7 \mathrm{La}$ idea de la incorporación de los procesos de gobernanza la extraen del modelo de ${ }_{3} \mathrm{P}$ (potencial, performance, procesos) de competitividad propuesto por Buckley, Pass y Prescott (1988). Para estos autores, la competitividad a cualquier nivel de análisis (firma, industria, país) puede ser medida por el potencial competitivo, el desempeño (performance) competitivo y el proceso competitivo.

8 Vale mencionar que los factores mencionados por Kresl que conforman los determinantes estratégicos, se pueden asimilar a lo que Deas y Giordano (2001) denominan "gobernanza".

${ }_{9}$ Kresl y Singh (1995), referenciado en Kresl y Singh $(1999,2012)$.

10 Trabajadores EARM = Ingenieros (Engineering), Contadores (Accounting), Investigadores (Researchers) y Gerentes (Management).

${ }_{11}$ Esta presunción va en línea con la discusión realizada en la sección anterior sobre los cuidados necesarios que hay que tener en cuenta al realizar comparaciones entre ciudades distintas.

12 Este acercamiento metodológico fue usado por Leal (2015) para la aplicación al caso del Estado de Querétaro en México (competitividad a nivel de Estado).

${ }_{13}$ En secciones previas, se mencionó que existían dos tipos de estudios que abordaban el tema de la competitividad y su medición. Los estudios de carácter académico componen uno de estos grupos y fueron en los que se centró la revisión de antecedentes.
} 